\title{
Artemis: A Reusable Excursion Vehicle Concept for Lunar Exploration
}

\author{
David A. Young ${ }^{*}$, John R. Olds ${ }^{\dagger}$, Virgil Hutchinson ${ }^{*}$, Zachary Krevor ${ }^{*}$, James Young* \\ Space Systems Design Lab \\ Guggenheim School of Aerospace Engineering \\ Georgia Institute of Technology, Atlanta, GA, 30332-0150 \\ david_young@ae.gatech.edu
}

\begin{abstract}
Artemis is a reusable excursion vehicle for lunar landing missions. It is intended to transport a notional CEV vehicle from low lunar orbit (LLO) to the lunar surface. It can be reused by refueling the vehicle in LLO. Artemis is nominally sized to carry a 10 MT payload to the lunar surface and then return it to LLO.

Artemis is powered by four liquid oxygen and liquid hydrogen fueled RL-10 engines. These RL-10 engines provide the necessary thrust and allow the Artemis lander to complete its nominal mission with two engines inoperative. The Artemis lander has volume margin built into its propellant tanks. This volume margin combined with an innovative cross-feed system allows Artemis to complete its ascent from the lunar surface with a propellant tank failure. This cross-feed system also allows Artemis to adjust the center of gravity (cg) of the vehicle by transferring propellant among the propellant tanks. Artemis lands on the moon with six articulating legs. This provides redundancy against a leg failure on landing and provides Artemis with the ability to land on uneven terrain.

This vehicle is designed to be launched by a heavy-lift evolved expendable launch vehicle (EELV). This design constraint results in the distinct shape of the lander. Artemis is launched as a compact cylinder in the EELV payload shroud, and then autonomously assembles itself via robotic arms similar to those currently used by the shuttle program.

Details of the conceptual design process used for Artemis are included in this paper. The disciplines used in the design include configuration, propulsion design and selection, trajectory, mass properties, structural design, cost, operations, and reliability and safety. Each of these disciplines was computed using a conceptual design tool similar to that used in industry. These disciplines were then combined into an integrated design team process and used to minimize the gross weight of the Artemis. Once the design process was completed, a parametric Excel based model was created from the point design. This model can be used to resize Artemis for changing system metrics (such as payload) as well as changing technologies.

The Artemis recurring and non-recurring costs were also computed. The total development cost including the design, development, testing and evaluation (DDT\&E) cost is \$2.17 B FY'04. The theoretical first unit (TFU) cost is \$303 M FY'04. Trade studies on life cycle costs (LCC) vs. fuel cost to LLO as well as flight rate are also discussed. A summary of design disciplines as well as the economic results are included.
\end{abstract}

\section{Nomenclature}

$C A D=$ computer aided design

CER = cost estimating relationship

${ }^{*}$ Graduate Research Assistant, School of Aerospace Engineering, Student member AIAA.

${ }^{\dagger}$ Associate Professor, School of Aerospace Engineering, Associate Fellow AIAA.

Copyright (C) 2005 by David Young and John R. Olds. Published by the American Institute of Aeronautics and Astronautics, Inc. with permission. 


$\begin{array}{ll}C E V & =\text { crew exploration vehicle } \\ D D T \& E & =\text { design, development, test, \& evaluation } \\ D S M & =\text { design structure matrix } \\ E E L V & =\text { evolved expendable launch vehicle } \\ E T O & =\text { Earth to orbit } \\ F E A & =\text { Finite Element Analysis } \\ \text { Isp } & =\text { specific impulse, sec } \\ L C C & =\text { life cycle cost } \\ L E O & =\text { low earth orbit } \\ L H 2 & =\text { liquid hydrogen } \\ \text { LLO } & =\text { low lunar orbit } \\ L O X & =\text { liquid oxygen } \\ M E C O & =\text { main engine cutoff } \\ M E R & =\text { mass estimating relationship } \\ M R & =\text { mass ratio (gross weight } / \text { burnout weight) } \\ N A F C O M & =\text { NASA Air Force Cost Model } \\ O E I & =\text { one engine inoperative } \\ T F U & =\text { theoretical first unit }\end{array}$

\section{Introduction}

A rtemis is a new lunar excursion vehicle designed to meet NASA's requirement for a lunar excursion vehicle to meet the national vision for space exploration. Artemis is a single-stage, man-rated, lunar excursion vehicle designed to ferry the manned CEV to the lunar surface. The emphasis on safety and cost has driven the Artemis design. To mitigate the risks (both cost and reliability) associated with the development of new components and technologies Artemis utilizes flight proven hardware whenever possible. Artemis builds on current propulsion technologies including four RL-10 derivatives for main propulsion. The propellant tanks and associated plumbing are made of shuttle derived materials, which takes advantage to the tremendous amount of knowledge currently available in manipulating these materials.

Due to the emphasis on crew safety, Artemis is designed with four RL-10s. These four engines allow for two engines to fail before the mission success is compromised. The Artemis lander has volume margin built into its propellant tanks. This volume margin combined with an innovative cross-feed system allows Artemis to complete its ascent from the lunar surface with a propellant tank failure. This cross-feed system also allows Artemis to adjust the center of gravity (cg) of the vehicle by transferring propellant among the propellant tanks. Artemis lands on the moon with six articulating legs. This provides redundancy against a leg failure on landing and provides Artemis with the ability to land on uneven

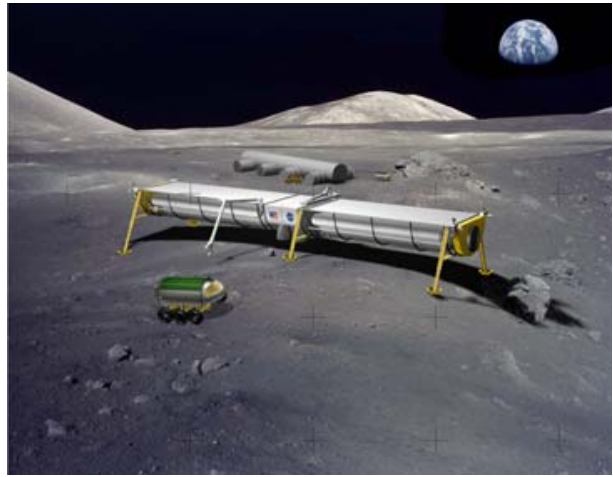

Figure 1. Artemis on Lunar Surface. terrain. This redundancy results in a reliability of 0.996 for Artemis.

The only new technologies necessary for the Artemis design is the autonomous assembly in LEO and the autonomous rendezvous and docking. These technologies are more software driven than hardware and although provide some risk to the program, are necessary for most vehicles in the lunar architectures. This risk will therefore need to be addressed by the entire lunar architecture and not just the excursion vehicle.

Artemis addresses the need for a lunar excursion vehicle with an innovative single-stage design. This one stage allows for reuse of the main hardware. This reuse has the advantage that a single launch of hardware can meet the excursion needs of multiple lunar missions. The engine out capability, cross-feed system, and six leg design, address the need for redundancy in a system that needs to have a high reliability (due to the reuse and fact that the vehicle is crewed). The single stage vehicle also has the advantage of being launched on a single EELV-H. This one launch of hardware can address the excursion needs of multiple lunar missions.

The nominal payload for the Artemis lander is $10 \mathrm{MT}$ to the surface and $10 \mathrm{MT}$ returned to LLO. This payload is meant to carry a nominal wet CEV. This can be either an inspace transfer module or the actual crewed launch 
vehicle. The modularity of the payload deck allows the Artemis lander to be used for both crewed CEV landings as well as cargo resupply missions to the surface.

A multi-disciplinary conceptual design process is used to create the Artemis design. This design process was completed using a disciplinary design tool for each of the following disciplines: external configuration and CAD was completed using ProEngineer, propulsion design and selection was completed using REDTOP ${ }^{1}$, trajectory optimization used POST $^{2}$, mass estimation and sizing was completed using mass estimating relationships ${ }^{3}$ (MERs), Cost estimating was conducted using NAFCOM cost estimating relationships (CERs), and reliability was completed using Relex ${ }^{4}$. Each of these tools was used to analyze their respective disciplines and was iterated to close the Artemis lander.

\section{Artemis Configuration}

Artemis is a single stage lunar lander derived from existing flight hardware to meet NASA's requirements for lunar excursion missions. To meet the NASA requirements Artemis was designed to be both highly reliable as well as light enough to be launched on an existing EELV. Artemis consists of two identical half cylindrical structures encasing six propellant tanks (four LOX and two larger LH2). Artemis has four RL-10 A-6 engines mounted on a movable platform at the center of the two halves of the Artemis structure. This placement of the engines allows for control of the vehicle in the event that an engine is lost. Artemis has six articulating legs. These legs are designed to accommodate landing on uneven terrain ( 1 meter clearance between surface and end of engine nozzle), as well as accommodating a leg failure. There are also two robotic arms attached to each half of the main structure. These arms serve two purposes. The first is to aid in the automated assembly of the Artemis vehicle in LEO. The

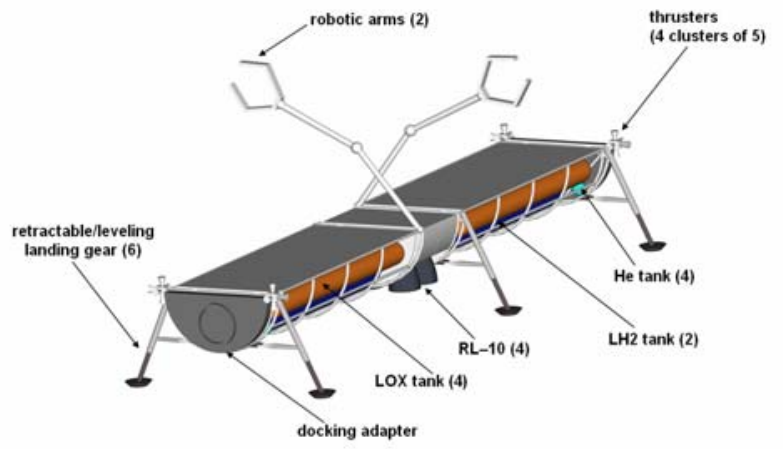

Figure 2. Artemis Configuration. second function is to help in loading the vehicle on the surface of the moon.

The single stage design of the Artemis allows the lander to be completely reusable. This gives Artemis a significant advantage in total launch weight necessary for a sustained lunar mission. Due to the extreme weight constraints with both being reusable and launching on an EELV-H, liquid oxygen and liquid hydrogen are used as the propellants. These propellants result in high efficiency as well as commonality to existing launch vehicles. Due to the volatility of storing these propellants for long term in lunar orbit each lunar mission will require a propellant resupply.

Artemis is designed to carry $10 \mathrm{MT}$ of payload from a $100 \mathrm{~km}$ circular low lunar orbit, and then return that same payload to the $100 \mathrm{~km}$ orbit. This payload class of $10 \mathrm{MT}$ is a rough estimate for the weight of an inspace transport vehicle for four crew. Artemis provides both the propulsion as well as the power necessary for the CEV during its transit to the lunar surface. Some consumables will be left on the surface and the resulting weight difference between the landing payload and the ascent payload can be made up with lunar samples.

Reliability and safety are main concerns for manned vehicles. Artemis is designed to accommodate two engine failures on any point of the trajectory both to and returning from the surface. As noted earlier legs also carry redundancy. A final margin has also been added to the tank volume. The modular design of the tanks, as well as a crossfeed system allows the Artemis lander to sustain a tank failure on ascent and still meet its reference orbit of 100 $\mathrm{km}$ circular. This redundancy and margin allows the reliability of the Artemis lander to approach 1 failure in 300 flights.

In the design of Artemis very few advanced technologies were assumed. Artemis assumes almost no hardware advances other than a highly throttleable variant of the RL-10 denoted the RL-10 A6. Most of the required technological advances are in the software and operations realm. Artemis assumes that unmanned on-orbit assembly of the vehicle can be accomplished. Artemis also assumes that some sort of advanced rendezvous and docking exists for mating with the CEV. These advances will be necessary with any sustained lunar mission and are assumed to be existing by the time of the Artemis deployment. As noted, Artemis design takes advantage of current propulsion developments such as the RL-10 family of engines. 
Artemis is designed to fit within the payload fairing of the EELV-H. This has driven the distinct shape of Artemis. The two half cylindrical structures make up the main portion of the vehicle. These half cylinders can fold to fit inside the existing EELV fairing. The engines are situated on their own preassembled platform. This square platform consists of the entire propulsion system including all associated thrust structure and plumbing. This allows a minimal amount of fluid connections that have to mate in on-orbit assembly. The total length of Artemis is almost 29 meters. This large surface allows significant room for a CEV vehicle as well as any associated surface equipment necessary.
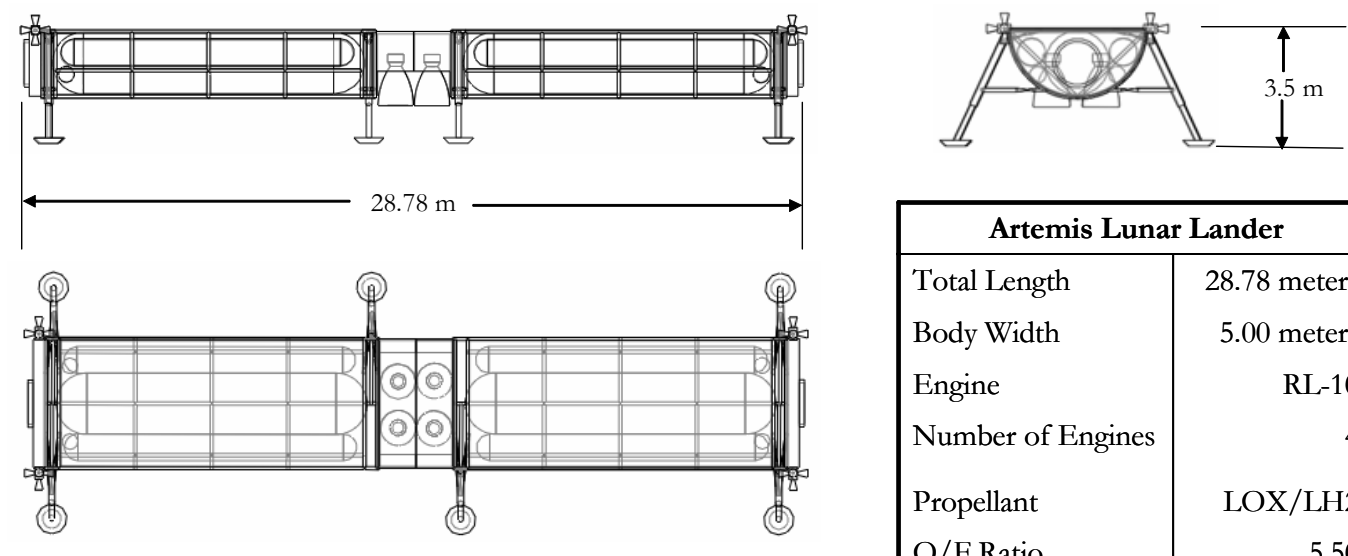

\begin{tabular}{|l|r|}
\hline \multicolumn{2}{|c|}{ Artemis Lunar Lander } \\
\hline Total Length & 28.78 meters \\
Body Width & 5.00 meters \\
Engine & RL-10 \\
Number of Engines & 4 \\
Propellant & LOX/LH2 \\
O/F Ratio & 5.50 \\
Lunar Payload & $10 \mathrm{MT}$ \\
\hline
\end{tabular}

Figure 3. Artemis Three-view Drawing.

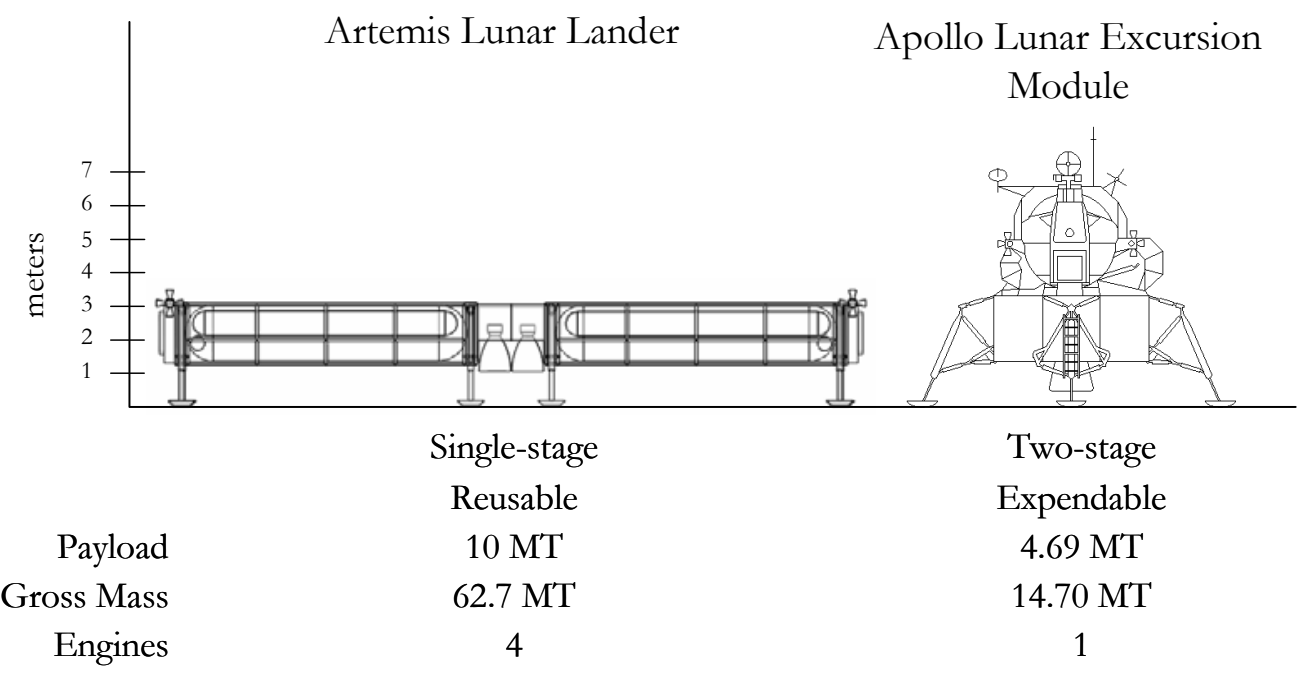

Figure 4. Comparison of Lunar Excursion Vehicles.

Artemis is comparable in size to the Apollo Lunar Excursion Module (LEM). The main differences between the two landers can be seen in Figure 4. The Apollo lander has lower ground clearance than the Artemis lander as well as single fault failures in both the engines and the landing legs. The main difference in height is due to the Apollo lander having the habitat permanently attached. The Artemis lander relies on the CEV for the life support systems necessary for the crew. The Artemis lander payload capacity is also double that of the Apollo LEM. This is necessary to carry the four man crew (vs. two for Apollo) to the surface. 


\section{Multidisciplinary Design Process}

The conceptual design process involves the combination of many different design disciplines. These disciplines are treated as individual contributing analyses to the entire vehicle design. Each of these contributing analyses are coupled which makes a difficult design problem. This coupling requires iteration between the disciplines to close the vehicle design. This coupling is graphically represented as a design structure matrix (DSM). Each of the contributing analyses (design disciplines) is represented as a box in the DSM and the links between the boxes are the coupling variables that are passed between the disciplines. Links leaving the right hand side of the boxes represent data that is passed downstream, while links leaving the left hand side represent information that is required upstream in the design process.

The DSM for the Artemis design involves two different iteration loops. The first iteration loop is between the propulsion, trajectory, and weights and sizing disciplines. This iteration loop closes the performance aspects of the vehicle. The second iteration loop is between operations, reliability, and cost. This loop uses the converged physical design and creates the operations, reliability, and costs of the closed design.

Each discipline has its own conceptual design tools associated with it. Table 1 provides a listing of each discipline and its associated design tool or tools. Configuration, propulsion, trajectory, and reliability are all analyzed with their respective disciplinary tool. Weights and sizing is composed of a series of MERs that are summarized and internally closed in an MS Excel workbook. Cost CERs are based upon NAFCOM and are also summarized in a MS Excel workbook.

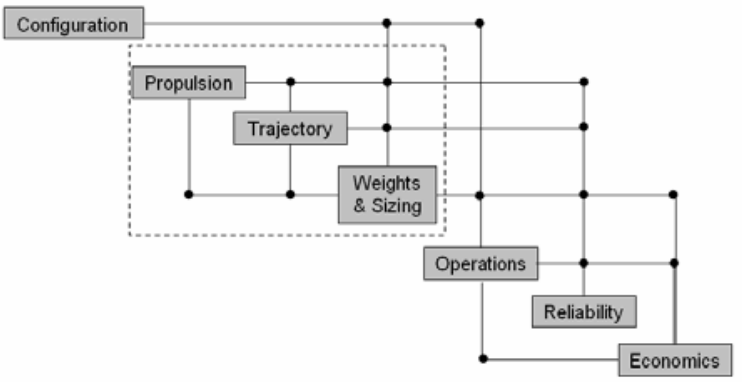

Table 1. Artemis Design Tools.

\begin{tabular}{cc}
\hline Discipline & Analysis Tool \\
\hline Configuration & Pro/E \\
Propulsion Design & REDTOP \\
Trajectory & POST 3-D \\
Weights \& Sizing & MS Excel \\
Reliability & Relex \\
Operations & AATE \\
Cost & NAFCOM \\
\hline
\end{tabular}

Figure 5. DSM for Artemis Design.

\section{Artemis Closure Results}

Each of the design disciplines depicted in Figure 5 are explained below in this section. Each of the design disciplines was completed and then iterated to achieve a closed Artemis design.

\section{A. Internal Configuration and Layout (CAD)}

The Artemis design consists of two identical structural components. These components commonality reduces the manufacturing complexity as well as allowing for simple on orbit assembly. The overall vehicle is approximately 29 meters long and 5 meters wide. This long rectangular design is a mainly due to the necessity that Artemis fit into an EELV-H fairing with a minimal amount of assembly required in orbit. 


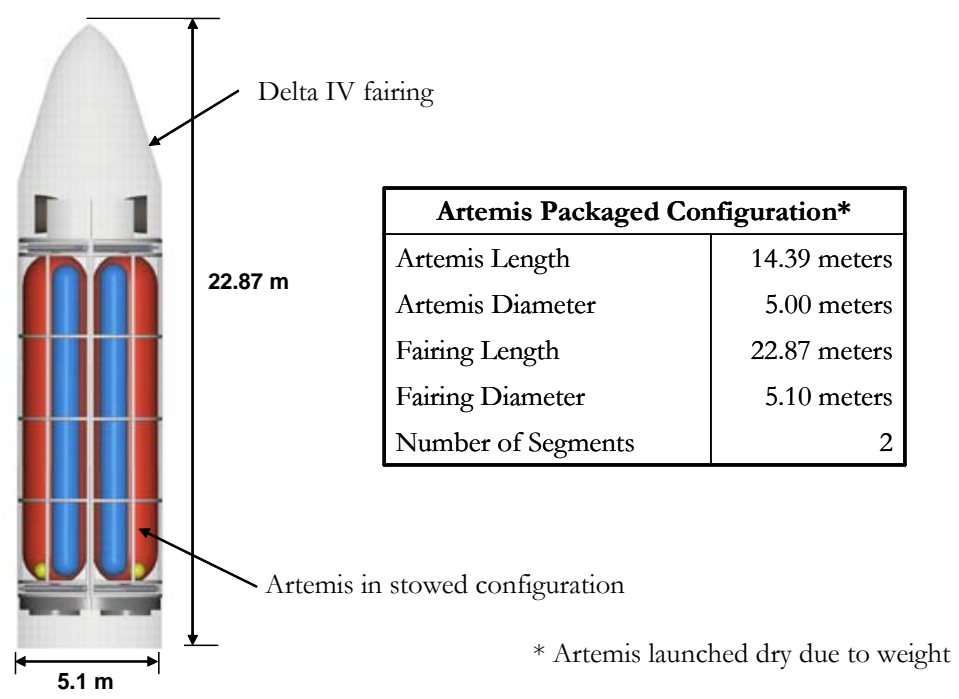

Figure 6. Artemis in Stowed Configuration.

Figure 6 depicts Artemis in its stowed configuration. This figure shows how only one assembly must be completed in orbit. This single assembly also only has only one fluid connection for the cross feed system. The excessive weight of the fully fueled vehicle as well as the complications of launching with fueled cryogenic vehicles forces Artemis to be launched dry. The propellant for the mission must be launched on future ETO missions.

The Artemis lander employs four RL-10s for propulsion. These main engines require liquid hydrogen and liquid oxygen for propellants. These propellants are stored in six tanks in the lander (two hydrogen and four oxygen). These tanks are also oversized to provide extra margin for propellant transfers to control the center of gravity during both descent and ascent. During the ascent phase this extra margin and crossfeed allows the Artemis lander to reach LLO even if one of the tanks fails.

The four RL-10s required for main propulsion are arraigned in a box formation in the center of the lander. As noted Artemis is design to complete its nominal mission with up to two engines failing. This center placement of the engines is chosen so that any engine failure does not result in a control problem. There are also multiple reaction control system engines on the corners of the lander. These RCS engines provide the precise control necessary for docking and on-orbit assembly.

\section{B. Propulsion Design}

The Artemis design uses two types of propulsion elements. The main engines are derivates of the RL-10 family. The engine chosen is derived from the RL-10 A5 and is denoted the A6. A derivate is necessary since the RL-10 does not provide the throttling necessary for the Artemis trajectory. This version of the RL-10 is designed using REDTOP $^{1}$, a conceptual chemical rocket design tool. The RL-10 is designed much like it's predecessors to be a $99 \mathrm{kN}(22,000 \mathrm{lb})$ thrust expander cycle engine. The Isp of this engine was designed at $449 \mathrm{sec}$. This Isp could be improved with a longer nozzle, but this was decided to be prohibitive due to the ground clearance necessary for a lunar landing on unfamiliar terrain. This engine is throttleable from $30 \%$ of its design thrust $(\sim 30 \mathrm{kN})$. The engine is also designed to be restartable multiple times without any refurbishment or replacement in the start cartridges. A summary of the RL-10 A6 performance is provided as Figure 7.

The reaction control system (RCS) engines are ISS derived RS-52 LOX/LH2 engines. Their low thrust and relative low weight allows them to be deployed in clusters at the corners of Artemis. These engines will be utilized for precision control of the lander. This is especially important for docking procedures as well as on-orbit assembly. A summary of the performance characteristics of the RS-57 (Figure 8) are shown below. 
- Expander Cycle

- LOX/LH2 Propellant

- $\mathrm{T}_{\mathrm{vac}}=99.2 \mathrm{kN}$

- $\mathrm{Isp}_{\mathrm{vac}}=449 \mathrm{~s}$

- Weight $_{\text {Installed }}=176 \mathrm{~kg}$

- Area $_{\text {exit }}=1.38 \mathrm{~m}^{2}$

- Expansion Ratio = 84

- $\mathrm{O} / \mathrm{F}$ Ratio $=5.5$

- Throttle Range $=30 \%-100 \%$

- $\mathrm{P}_{\text {chamber }}=3.206 \mathrm{MPa}$

- Reusable, multiple restarts

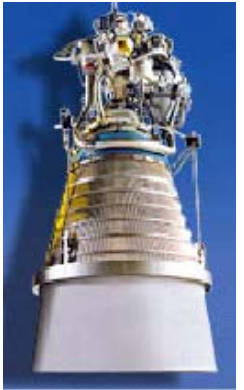

- LOX/LH2 Propellant

- $\mathrm{T}_{\mathrm{vac}}=0.1335 \mathrm{kN}$

- $\mathrm{Isp}_{\mathrm{vac}}=427 \mathrm{~s}$

- Weight $_{\text {Installed }}=11.34 \mathrm{~kg}$

- Area $_{\text {exit }}=1.05 * 10^{-2} \mathrm{~m}^{2}$

- Expansion Ratio $=100$

- $\mathrm{O} / \mathrm{F}$ Ratio $=6$

- $\mathrm{P}_{\text {chamber }}=0.69 \mathrm{MPa}$

Figure 7. RL-10 A-6 Engine Design Specifications.

Figure 8. RS-52 RCS Engine Design Specifications.

The RL-10 A6 could also be used for other lunar applications. This engine would be ideal for an inspace transefer vehicle or as the main propulsion in a power and propulsion module for the CEV.

\section{Performance}

The trajectory of the Artemis lander is optimized using a three degree of freedom trajectory simulation code called the Program to Optimize Simulated Trajectories (POST 3-D) ${ }^{2}$. As noted earlier in this paper the Artemis payload requirement of $10 \mathrm{MT}$ is used to size the vehicle, while the constraint of the 5 meter payload fairing is used to limit the overall size.

The Artemis descent and ascent trajectories are optimized to minimize the gross weight of the vehicle by changing the pitch angles. The constraints on the ascent and descent trajectories are: the initial orbit, the final orbit (100km circ.), the $\mathrm{g}$ forces for the trajectories must not be greater than $3 \mathrm{~g}$ 's, and the final payload must be $10 \mathrm{MT}$. The trajectory also has a built-in 30 second hover for margin and safety requirements.

As shown in Figures 9 and 10, the Artemis trajectories both begin and end at the same $100 \mathrm{~km}$ circular orbit. In the descent trajectory an ideal delta V burn is used to put the vehicle in a $0 \mathrm{X} 100 \mathrm{~km}$ orbit. The vehicle then begins the descent to the moon. In Figure 9 it is shown that the altitude decreases and the velocity increases as the lander is basically falling to the moon. POST is allowed to determine the ideal point to fire the engines. At approximately 2,350 seconds into the descent the engine cluster begins to fire and slow the vehicle's descent considerably. This allows for a soft $(<0.5 \mathrm{~m} / \mathrm{s})$ landing on the surface of the moon.

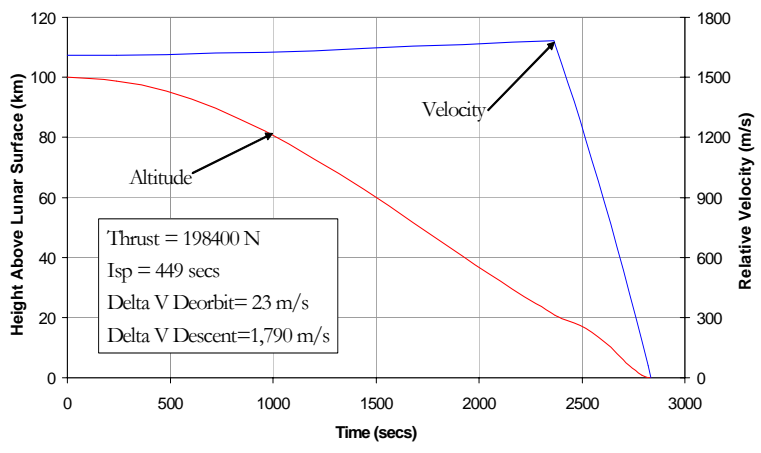

Figure 9. Descent Trajectory.

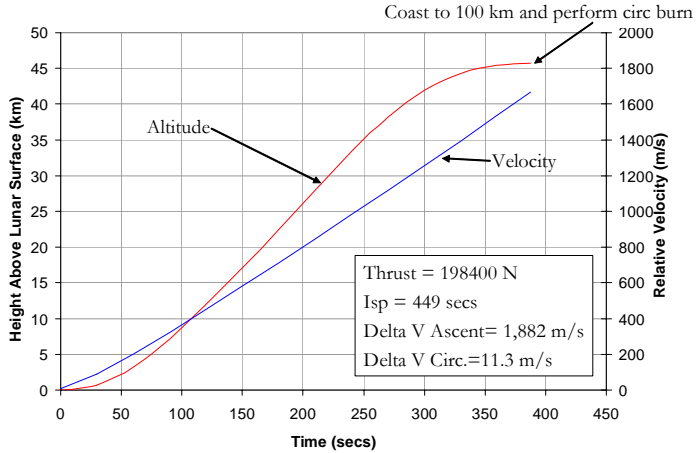

Figure 10. Ascent Trajectory.

The ascent trajectory is shown in Figure 10. As this figure shows the Artemis lander begins it ascent from the surface and travels to an orbit that has an apoapsis of $100 \mathrm{~km}$. The periapsis of this orbit is optimized between POST and the propellant necessary for the ideal delta V burn necessary to circularize the orbit at $100 \mathrm{~km}$. 


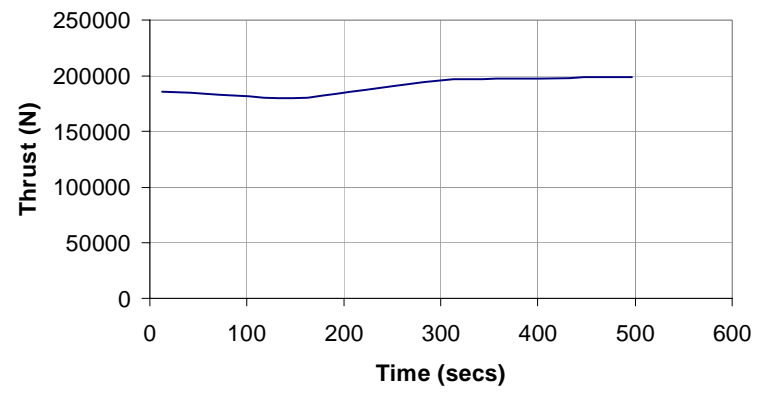

Figure 11. Descent Thrust from Point of Engine

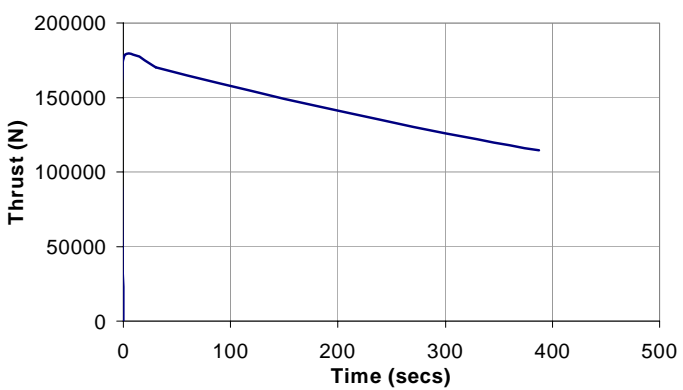

Figure 12. Ascent EngineThrust.

Ignition ( 2350 secs).

These figures (11 and 12) show the powered phase of flight. As figure 11 shows the engines throttle down in the beginning of the descent profile to save fuel. The max thrust is only needed as the vehicle approaches the surface. Figure 12 shows the Ascent thrust profile. As this figure shows the max thrust of the engines is only needed at the surface of the moon. As the velocity increases the engines have to throttle down to avoid violating the maximum acceleration constraint.

\section{Mass Estimation \& Structural Design}

Mass estimation is computed using MERs for each of the components of the Artemis lander. The vehicle weight is broken into twelve categories. The MERs are based upon the required volume for propellants as well as tank and structural weights. The mass properties spreadsheet adjusts the size and weight of the vehicle to match the propellant output from the trajectory analysis. As the required propellant increases the spreadsheet increases dry weight of the vehicle by sizing the tanks to match the new required propellant load. The subsystems and interstages also parametrically scale with the dry weight. The propellant and dry weight are then iterated on between the trajectory and weights disciplines until the design is closed. A summary of the closed weights for Artemis are included as Figure 13.

\begin{tabular}{cc}
\hline Artemis Lunar Lander & \\
\hline 1.0 Body Group & $8.79 \mathrm{MT}$ \\
LOx Tanks & $2.22 \mathrm{MT}$ \\
LH2 Tanks & $2.21 \mathrm{MT}$ \\
He Tanks & $0.19 \mathrm{MT}$ \\
Other Structure & $4.17 \mathrm{MT}$ \\
2.0 Main Propulsion & $1.02 \mathrm{MT}$ \\
3.0 RCS Propulsion & $0.08 \mathrm{MT}$ \\
4.0 Primary Power & $0.57 \mathrm{MT}$ \\
5.0 Electrical Conversion and Distribution & $0.08 \mathrm{MT}$ \\
6.0 Avionics & $0.50 \mathrm{MT}$ \\
7.0 Margin & $1.66 \mathrm{MT}$ \\
Dry Weight & $12.70 \mathrm{MT}$ \\
8.0 Payload to LLO & $10.00 \mathrm{MT}$ \\
Docking (Return) Weight & $22.70 \mathrm{MT}$ \\
9.0 Ascent Propellant & $13.50 \mathrm{MT}$ \\
LOx & $11.42 \mathrm{MT}$ \\
LH2 & $2.08 \mathrm{MT}$ \\
Ascent (Takeoff) Weight & $36.20 \mathrm{MT}$ \\
10.0 Payload to LS & $10.00 \mathrm{MT}$ \\
Landing Weight & $36.20 \mathrm{MT}$ \\
11.0 Descent Propellant & $25.27 \mathrm{MT}$ \\
LOx & $21.38 \mathrm{MT}$ \\
LH2 & $3.89 \mathrm{MT}$ \\
RCS propellant & $0.50 \mathrm{MT}$ \\
12.0 Startup Losses & $61.46 \mathrm{MT}$ \\
Gross Weight & $0.25 \mathrm{MT}$ \\
Outbound Weight & $61.72 \mathrm{MT}$ \\
\hline
\end{tabular}

Gross Weight Breakdown

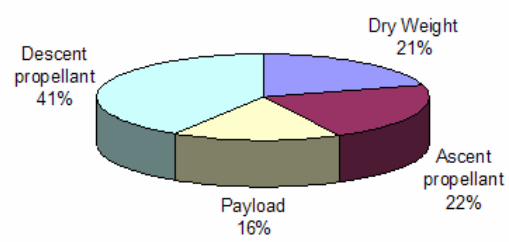

Dry Weight Breakdown

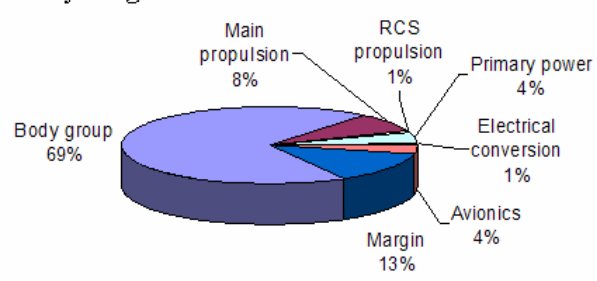

Figure 13. Artemis Mass Summary 
As shown in Figure 13 the gross weight of the lander is 62 MT, with a dry weight of 12.7 MT. This dry weight includes a $15 \%$ design margin. The total gross weight includes all the propellant necessary for the mission as well as the 10MT payload. The descent delta V is very similar to the ascent delta V. The main difference in propellant weight is the fact that the ascent propellant must be carried to the surface and therefore the descent propellant used is much greater than the ascent propellant. The biggest contributor for the dry weight of the vehicle is the tanks and other structure such as legs and engine mountings. The two engine out capability of the vehicle results in a slight weight penalty for carrying the extra engines. This weight penalty results in approximately $500 \mathrm{~kg}$ of extra mass.

A structural analysis of the legs using a finite element analysis (FEA) was completed. This was used to determine if the leg weight to withstand the $1 \mathrm{~m} / \mathrm{s}$ landing speed. The legs are designed from an aluminum honeycomb structure with an overall load of $8175 \mathrm{~N}$ per leg. The resulting leg weight is $208 \mathrm{~kg}$ per leg. A structural analysis is shown in Figure 14.

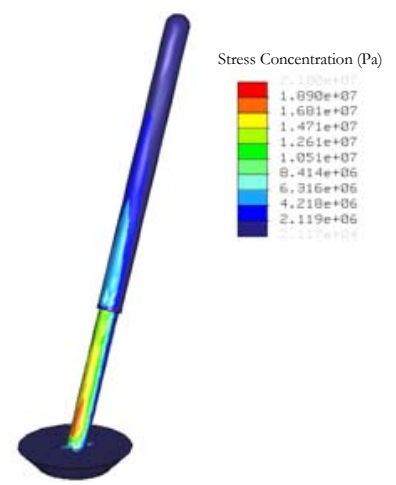

Figure 14. FEA of Artemis

\section{Landing Gear.}

\section{E. Reliability}

The Artemis reliability is calculated using Relex ${ }^{4}$, a fault tree analysis Software product. The reliability of each of the subsystems is inputted into Relex and the resulting reliability of the system can be obtained. For this analysis, an improvement in the historic reliability of ELV subsystems as well as avionics, structure, and propellant storage is assumed. These improvements can be attributed to the increased emphasis on safety currently employed in space vehicle design. It is also assumed that the RL-10 has a reliability of 1 failure in 4000 starts. A summary of the Relex analysis is included as Figure.

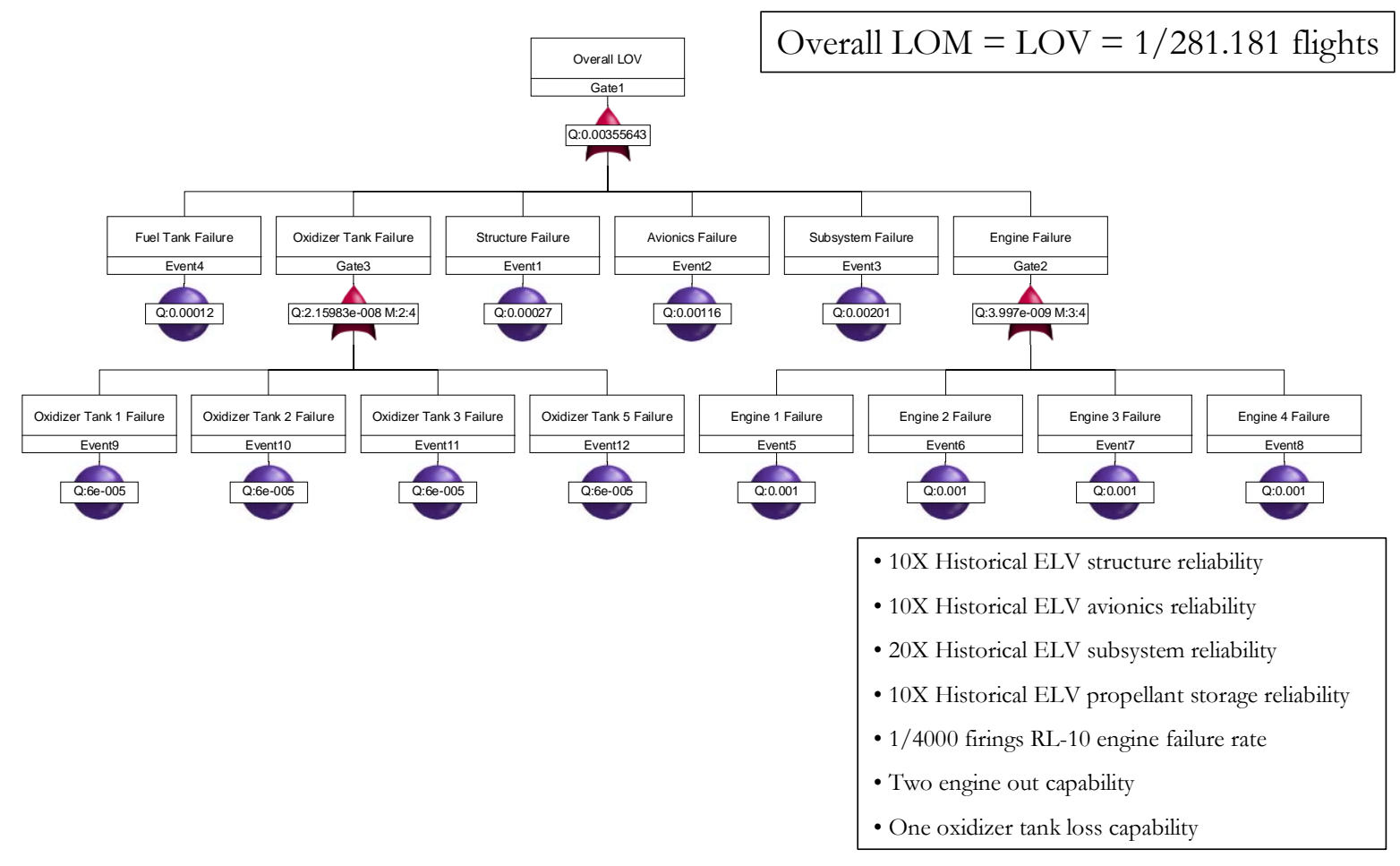

Figure 15. Reliability Analysis with Relex. 
As this analysis shows even with the improvements on historical ELV subsystems and engine components the overall reliability of the Artemis design is one failure in 281 flights or a 0.996 reliability. This high reliability is necessary for a manned lunar lander since an abort is highly unlikely so far from the Earth. Also the reusability of this vehicle requires it to accomplish multiple missions. A loss of vehicle could jeopardize the entire lunar program and therefore must be avoided.

\section{F. Cost and Economics}

The cost estimating for the Artemis design is calculated using weight-based cost estimating relationships (CERs). These CERs are used to estimate the development and production costs for each of the items in the weight breakdown structure. These CERs are based upon data from the NASA Air Force Cost Model (NAFCOM) for cost estimating. For this cost analysis it is assumed that Artemis is infinitely reusable for the 20 year life span of the program. Also it is assumed that the design, development, testing and evaluation (DDT\&E) costs will be spread evenly over the 20 years of the lunar program. A summary of the design, development, testing and evaluation costs (DDT\&E) as well as the production costs for the Artemis lander are included as Figure 16 (All costs presented in FY 2004 dollars).

The total vehicle cost to first unit (DDT\&E plus production costs) is calculated to be 2,486 $\$$ M. This can be broken down into $\$ 1.8 \mathrm{~B}$ for the DDT\&E of the airframe, \$378 $\mathrm{M}$ for the DDT\&E of developing an RL10 derivative, and $\$ 303 \mathrm{M}$ for the first (and only) production unit. As shown the biggest contributor to this cost is the high airframe DDT\&E. This high DDT\&E is

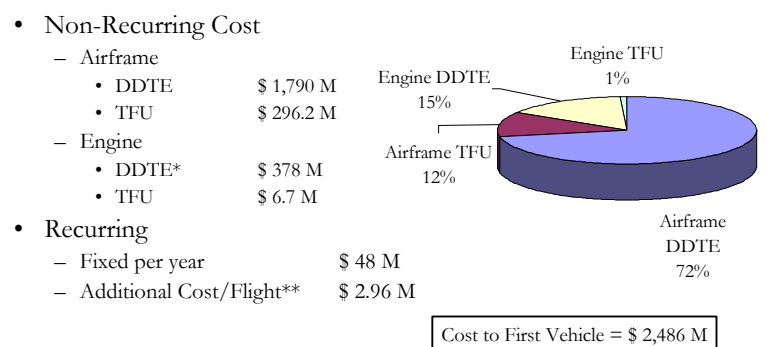
due to that fact that Artemis must be highly reusable and reliable. There is also a margin included in the cost calculations. This margin is $20 \%$ of the total vehicle costs and is used to approximate for unforeseen cost overruns.

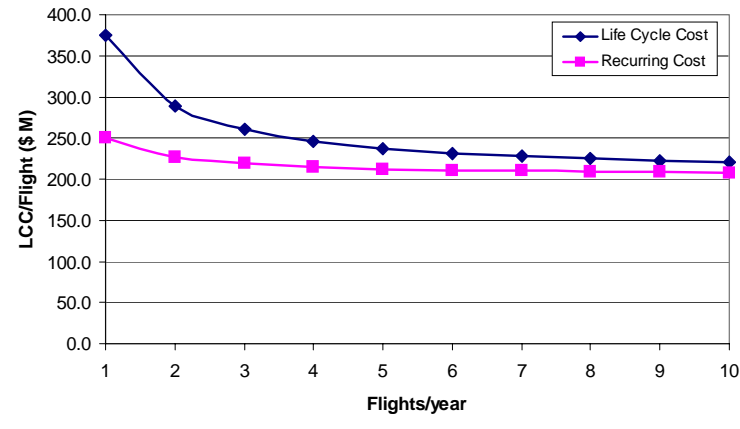

Figure 17. Artemis Cost Sensitivity to Flight Rate.

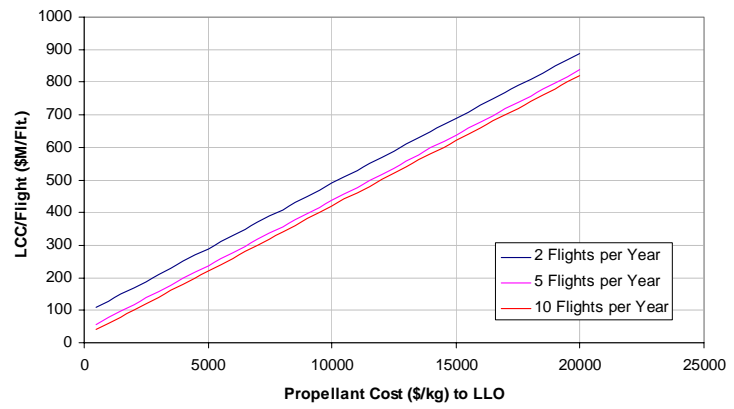

Figure 18. Artemis Cost Sensitivity due to Propellant Costs in LLO.

The life cycle cost (LCC) for the Artemis design is calculated based upon the flight rate and is shown in Figure 17. The LCC includes the initial investment (DDT\&E), the production and integration of airframe and engines, as well as a $\$ 5000 / \mathrm{kg}$ cost of propellant delivery to LLO. The LCC does not include the launch costs associated with launching the hardware or the propellant. The initial investment of \$2.486 B is spread evenly over the twenty year life of the program. The total undiscounted cost per flight is approximately $\$ 289 \mathrm{M}$ for the flight rate of two flights per year. As the flight rate increases the costs drop significantly as the initial investment is spread over all of the flights. The cost asymptote at about $\$ 200 \mathrm{M}$ as the flight rate approaches 10 flights per year.

The total cost of the Artemis program is very sensitive to the cost to deliver propellant in LLO. This cost can very widely from very aggressive assumptions involving lunar resource utilization to a very conservative assumption of conventional launch and deploy of propellant. The sensitivity of this cost of propellant as well as 
flight rate is shown in Figure 18. For the baseline an aggressive assumption of $\$ 5000 / \mathrm{kg}$ was chosen, if this cost were to double the total LCC/flight would increase by $70 \%$.

\section{Parametric Model}

After the design of the baseline, a parametric model of the Artemis excursion vehicle was completed to address the changing requirements for the design. The need to explore the design space quickly requires that the disciplinary tools are approximated for using well known first cut approximations. Configuration was scaled photographically to accommodate payload changes as well as mission changes such as time of flight and duration on the surface. The weights and sizing model was created using the same MERs used for the baseline as well as including some simple propulsion approximations for the number of necessary engines. The trajectory analysis was reduced to a delta velocity approximation with the rocket equation providing the needed propellant as the vehicle scaled. This resulted in a model that was accurate for the baseline case and scaled appropriately for a range of payloads from 5-30 MT and multiple changing mission requirements.

\section{Conclusions}

Artemis is a reusable excursion vehicle for lunar landing missions. It is intended to transport a notional CEV vehicle from low lunar orbit (LLO) to the lunar surface. Artemis is nominally sized to carry a 10 MT payload to the lunar surface and then return it to LLO. Artemis consists of two cylindrical half shell structural elements with four liquid oxygen and liquid hydrogen fueled RL-10 derivative engines. This configuration allows the Artemis lander to be launched by a heavy-lift EELV.

Artemis was designed with an emphasis placed on reliability. The four RL-10 engines provide the necessary thrust and allow the Artemis lander to complete its nominal mission with two engines inoperative. The Artemis lander has volume margin built into its propellant tanks. This volume margin combined with an innovative crossfeed system allows Artemis to complete its ascent from the lunar surface with a propellant tank failure. This crossfeed system also allows Artemis to adjust the center of gravity (cg) of the vehicle by transferring propellant among the propellant tanks. Artemis lands on the moon with six articulating legs. This provides redundancy against a leg failure on landing and provides Artemis with the ability to land on uneven terrain. The resulting reliability for the Artemis vehicle is 0.996 .

The Artemis recurring and non-recurring costs were also computed. The total development cost including the design, development, testing and evaluation (DDT\&E) cost is \$2.17 B FY'04. The theoretical first unit (TFU) cost is $\$ 303 \mathrm{M} F Y^{\prime} 04$. Artemis costs are significantly affected by the cost to deliver propellant to LLO. These costs can

drive the total costs to the program. A doubling of the cost of propellant delivery from $\$ 5,000 / \mathrm{kg}$ to $\$ 10,000 / \mathrm{kg}$ results in an increase in to total LCC of $70 \%$.

\section{Acknowledgments}

This work was funded under the Advanced Technology and Life-cycle AnalysiS Tool (ATLAS) program at NASA Marshall SFC via a grant from the National Space Science and Technology Center. The authors would like to thank the members of the Space Systems Design Lab at the Georgia Institute of Technology.

\section{References}

${ }^{1}$ Rocket Engine Design Tool for Optimal Performance (REDTOP), Software Package, ver 1.3, Spaceworks Engineering Inc., Atlanta, GA, 2003.

${ }^{2}$ Powell, R.W., et. al., "Program to Optimize Simulated Trajectories (POST) Utilization Manual, Volume II, Version 5.2,” NASA Langley Research Center, Hampton, VA; Martin Marietta Corporation, Denver, CO., October 1997.

${ }^{3}$ Rohrschneider, R., "Development of a Mass Estimating Relationship Database for Launch Vehicle Conceptual Design," AE8900 Special Project, School of Aerospace Engineering, Georgia Institute of Technology, April 26, 2002.

${ }^{4}$ Relex Reliability Suite, Software Package, ver 2.2, Relex Software Corporation, Greensburg, PA, 2004. 